\title{
Efficient in-depth analysis and optimum design parameter estimation of MEMS capacitive pressure sensor utilizing analytical approach for square diaphragm
}

SUMIT KUMAR JINDAL ( $\sim$ sumitjindal08@gmail.com )

VIT University

ISHAN PATEL

VIT University

KRISH SETHI

VIT University

SIMRIT KAUL

VIT University

SREEKANTH P K

VIT University

Ajay Kumar

National Institute of Technology Jamshedpur

\section{Research Article}

Keywords: Capacitive pressure sensor, Computationally efficient, Normal mode, Optimal operation, Sensitivity, Square Diaphragm, Touch mode

Posted Date: August 20th, 2021

DOl: https://doi.org/10.21203/rs.3.rs-532583/v1

License: (c) (i) This work is licensed under a Creative Commons Attribution 4.0 International License. Read Full License 


\section{Efficient in-depth analysis and optimum design parameter estimation of MEMS capacitive pressure sensor utilizing analytical approach for square diaphragm}

\section{Declarations:}

Funding- Not Applicable.

Conflicts of interest/Competing interests -Not Applicable.

Availability of data and material- Not Applicable.

Code availability- Licensed Version of MATLAB has been used to generate plots.

Authors' contributions- Optional (Not Applicable).

\begin{abstract}
Capacitive pressure sensors have become more popular as compared to piezoresistive pressure sensors as they yield superior sensitivity and lesser non-linearity. Efficient analysis for modelling capacitive pressure sensors is thus increasingly becoming more important due to their innumerable use cases. The higher sensitivity of square diaphragm for the same side length in comparison to circular diaphragm makes it ideal for sensor design. In this work, a complete formulation for analysis of capacitive pressure sensor with the square diaphragm in normal and touch mode operation has been presented as these two modes are established operating modes for these sensors. A comprehensive study of sensor parameters like capacitance, diaphragm deflection, capacitive and mechanical sensitivity has been formulated to aid the choice of sensor characteristics. This work also focuses on the method to determine core design parameters for optimal operation. Computationally complex methods have been used in the past for analysis of square diaphragms. In contrast to the finite element system, the analytical technique proposed in this study is less complex and computationally efficient (FEM). The results were computed and simulated using MATLAB.
\end{abstract}

Index Terms- Capacitive pressure sensor, Computationally efficient, Normal mode, Optimal operation, Sensitivity, Square Diaphragm, Touch mode

\section{INTRODUCTION}

Capacitive pressure sensors based on MicroElectroMechanical Systems (MEMS) play a critical role in MEMS devices for real-world scenarios ${ }^{[1]}$. This sensors have a long history of use in medical research, the automotive industry, avionics, and other industrial and commercial applications $[2][3]$. As a result of their mission-critical design, these pressure sensors must be well-designed and dependable when used in harsh environments. High sensitivity, low temperature drift, and low noise can all be features of these instruments. Initially, piezoresistive pressure sensors were used, but capacitive pressure sensors (CPS) were eventually equipped. The CPS's higher sensitivity and lower nonlinearity were the driving forces behind the switch from piezoresistive to capacitive pressure sensors. Capacitive pressure sensors often have a significantly wider linear output range than resistive pressure sensors. Reliable performance is witnessed even in high temperature conditions and harsh environments ${ }^{[4]}$.

These sensors use ceramic or silicon diaphragms and can operate in two modes, the normal mode, and the touch mode.
In comparison to normal mode operation, touch mode delivers near-linear output characteristics and large over range pressures and this has led to the design of Single Touch Mode Capacitive Pressure Sensor (STMCPS) with circular diaphragm ${ }^{11]}$. To further enhance performance, Double-sided Touch Mode Capacitive Pressure Sensor (DTMCPS) were proposed. In this design, an additional notch was etched at the bottom of the substrate providing higher linearity and enhanced sensitivity compared to STMCPS [2][3]. By etching two TMCPSs back to back in one substrate, a novel enhancement to both TMCPS and DTMCPS has been proposed. This structure was called the Double-sided Touch Mode Capacitive Pressure Sensor $(\mathrm{DSTMCPS})^{[4][5]}$. This enriched design provided exceptional results for differential pressure applications but entailed increased manufacturing and design costs $\frac{[6][7][8]}{\text {. Due to its }}$ outstanding electrical, electronic, and chemical attributes of high stability, conductivity, inertness, high critical electric field, hardness, resilience to wear and tear, and mechanical ruggedness silicon carbide ( $\mathrm{SiC}$ ) has emerged as an attractive component for high applications ${ }^{[4][9] .}$ Thus further innovations for circular diaphragms based CPS were brought about by using a sandwich structure composed of $\mathrm{SiC}$ and aluminium nitride $(\mathrm{AlN})$ for sensor construction. ${ }^{[10][11]}$

Square diaphragm-based CPS are more responsive to variations in applied pressure for a given side-length than their circular counterparts [6][9]. In addition, square diaphragms are preferred due to ease in fabrication and better yield [12]. Previous work shows the mathematical modelling for square diaphragms CPS employs highly involved and complex mathematical methods, however these techniques are computationally expensive [8][13][14]. An analytical solution for normal mode operation of CPS based on square diaphragms has been illustrated in ${ }^{[6]}$. As touch mode capacitive pressure sensors offer better performance in many applications, there is a need for efficient analysis of touch mode operation. This work therefore elucidates an indepth, step-by-step derivation of the capacitance, capacitive and mechanical sensitivity of a silicon-based CPS with square diaphragm in both normal and touch mode operation. An approach for choice of sensor dimensions for optimum design has been provided using MATLAB simulations. The analytical solution provided is based on small deflection theory and assumes linear elastic deformation of the square diaphragm. The model developed is efficient and eliminates 
the need for computationally exhaustive finite element method (FEM) modelling $\stackrel{[15][16]}{ }$.

The below is how the paper is structured: The theoretical model is discussed in the second part for analysis of square diaphragm CPS and demonstrates the different operating modes of the sensor. The third section lays out a comprehensive, step-by-step evaluation procedure for the capacitance, capacitive sensitivity, and mechanical sensitivity for normal and touch mode operation. In sections four and five, the results, analysis, and comparing of different parameters for the exact configuration of a MEMS pressure sensor is addressed.

\section{THEORY}

A capacitive pressure curve (Fig.1) is used to examine the behavior of square diaphragm-based CPS. Fig.1 illustrates four operation zones normal, transition, touch, and saturation mode.

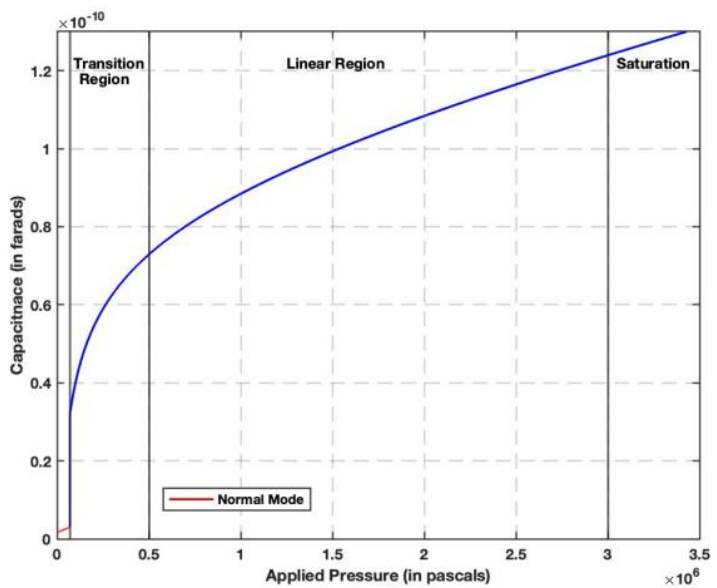

Fig. 1. Capacitance pressure curve describing the behavior of a square diaphragm-based CPS

During normal mode, the diaphragm does not touch the bottom electrode or the substrate which can be observed in Fig.2. The pressure range for normal mode spans from zero to the touch point pressure. The touch point pressure is the minimum pressure that is applied so that the diaphragm just touches the electrode. The sensor is said to be in transition mode as the plate reaches the bottom of the cavity, resulting in an extremely non-linear relationship between capacitance and pressure. The transition mode creates noise and hence is not utilized for sensor operation.

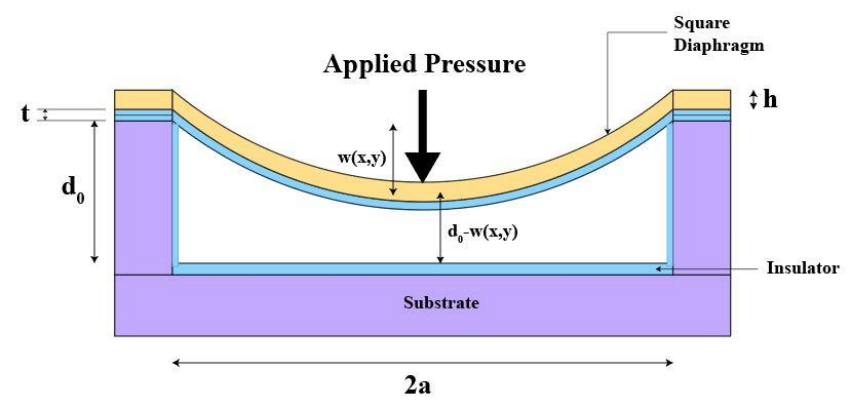

Fig. 2. Cross-sectional view of normal mode CPS
In touch mode operation of the device, the diaphragm makes a contact with the bottom electrode. This is depicted in Fig.3. An insulator layer is placed over the bottom electrode to prevent short circuit. The touch mode was introduced to enhance sensor performance. In saturation mode, the diaphragm completely touches the bottom electrode as a result, very less variation of capacitance takes place and ultimately capacitance saturates to a peak value.

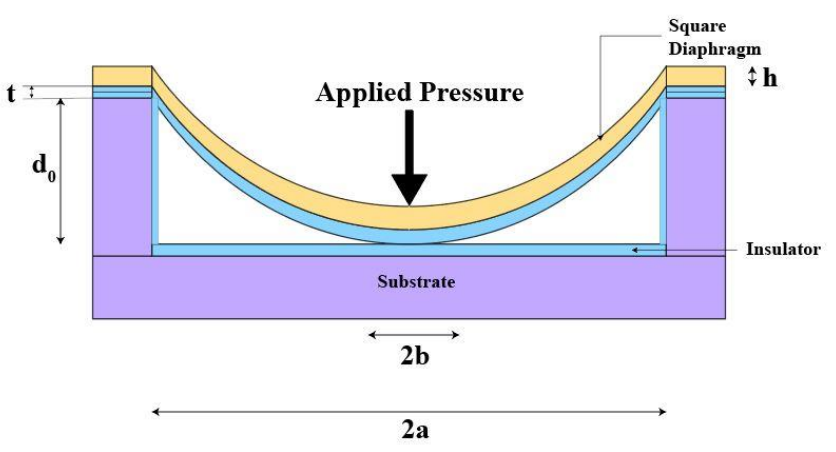

Fig. 3. Cross-sectional view of touch mode CPS

\section{ANALYTICAL SOLUTION}

The mathematical modeling presented in this work is based on the theory of small deflection for plate bending. According to the theory, diaphragm deflections are small in contrast to plate thickness.

Fig. 2 illustrates a square diaphragm of thickness $(h)$, Poisson's ratio $(v)$, Young's Modulus $(E)$ and side length (2a). The diaphragm deflection, $w(x, y)$ is a function of $x$ and $y$ co-ordinates given by 6$][16]$.

$w(x, y)=\frac{P}{47 D} \frac{\left(\left(x^{2}-a^{2}\right)^{2}\left(y^{2}-a^{2}\right)^{2}\right)}{a^{4}}$

Where $D$ is the flexural rigidity and can be defined as resistance offered by a non-rigid structure when it is undergoing bending, or when a force couple is applied to the structure ${ }^{[9][15][16]}$ and $P$ is the applied pressure. Flexural rigidity can be expressed as

$D=\frac{E h^{3}}{12\left(1-v^{2}\right)}$

The central deflection for square diaphragm can be evaluated by substituting $x=0$ and $y=0$ in (1)

$w_{0}=w(0,0)=w_{\text {center }}=\frac{P a^{4}}{47 D}$

The touch-point pressure is defined as the maximum pressure at which the square diaphragm just touches the surface of the lower electrode. At touch point pressure $(P=$ $p_{t}$ and $w_{0}=d_{0}$ ), where $d_{0}$ is the separation between the plates at zero deflection,

$p_{t}=\frac{47 D d_{0}}{a^{4}}$ 


\subsection{Normal mode capacitance analysis}

For a parallel plate capacitor, capacitance $C$ can be expressed as

$C=\frac{\varepsilon_{0} A}{d}$

where $\varepsilon, A$ and $d$ are the permittivity of gap, plate area and plate separation, respectively. This equation can be used to calculate normal mode capacitance by considering an elemental area and integrating the expression over the diaphragm dimensions. Hence, the expression for $C$ can be mathematically written as

$C=\int_{y=-a}^{a} \int_{x=-a}^{a} \frac{\varepsilon_{0}}{\left(d_{0}-w(x, y)\right)} \cdot d x \cdot d y$

Substituting the expression for $w(x, y)$ obtained in (1) in (6)

$C=\frac{\varepsilon_{0}}{d_{0}} \int_{y=-a}^{a} \int_{x=-a}^{a} \frac{1}{\left(1-\frac{P}{47 D} \frac{\left(\left(x^{2}-a^{2}\right)^{2}\left(y^{2}-a^{2}\right)^{2}\right)}{d_{0} a^{4}}\right)} \cdot d x \cdot d y$

Using the binomial approximation of

$\frac{1}{1-x}=(1-x)^{-1}=(1+x)$ for $x<<1$ in $(7)$

$C=\frac{\varepsilon_{0}}{d_{0}} \int_{y=-a}^{a} \int_{x=-a}^{a}\left(1+\left(\frac{P}{47 D} \frac{\left(\left(x^{2}-a^{2}\right)^{2}\left(y^{2}-a^{2}\right)^{2}\right)}{d_{0} a^{4}}\right)\right) \cdot d x . d y$

$C=\frac{4 a^{2} \varepsilon_{0}}{d_{0}}+\frac{\varepsilon_{0}}{d_{0}} \int_{y=-a}^{a} \int_{x=-a}^{a} \frac{P a^{4}}{47 D}\left(\frac{\left(1-\frac{x^{2}}{a^{2}}\right)^{2}\left(1-\frac{y^{2}}{a^{2}}\right)^{2}}{d_{0}}\right) \cdot d x . d y$

Assuming $C_{0}=\frac{4 a^{2} \varepsilon_{0}}{d_{0}},(9)$ is simplified as

$C=C_{0}+\frac{\varepsilon_{0}}{d_{0}} \frac{P a^{4}}{47 D d_{0}} \int_{y=-a}^{a}\left(1-\frac{y^{2}}{a^{2}}\right)^{2} d y \int_{x=-a}^{a}\left(1-\frac{x^{2}}{a^{2}}\right)^{2} d x$

On evaluating the integral in (10), $C$ can be expressed as

$$
C=C_{0}+C_{0} \frac{P a^{4}}{47 D d_{0}}\left(\frac{64}{225}\right)
$$

Substituting $p_{t}$ from (4) in (11), capacitance for normal mode operation is evaluated as
$C_{\text {normal }}=C_{0}+C_{0}\left(\frac{64}{225}\right)\left(\frac{P}{p_{t}}\right)$

\subsection{Touch mode capacitance analysis}

Touch mode contact for a square diaphragm CPS is illustrated in Fig. 3. Touch mode capacitance constitutes (1) touchdown capacitance of diaphragm $C_{t}$ and (2) cavity capacitance $C_{u t}^{[1]}$. $C_{t}$ can be evaluated using the concept of parallel plate capacitance. To solve for $C_{u t}$ axial symmetry of boundary condition approximation is used ${ }^{[1]}$. Electrical field flux lines can be approximated as directional arcs due to axis symmetry of the diaphragm and underlying substrate. This is shown in Fig. 4

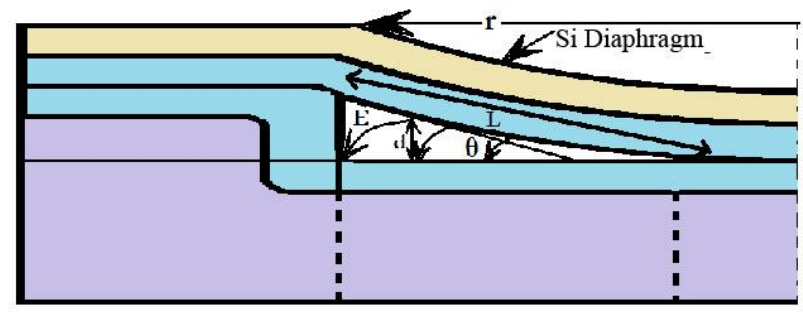

Fig. 4. Cross sectional view of electric flux lines between two electrodes

According to Gauss law,

$$
\rho=\vec{D}=\in \vec{E}
$$

where $\rho$ is total charge density, $D$ is electric displacement, $\epsilon$ is permittivity of medium. Considering electric flux as directional arcs, electric field intensity is

$E=\frac{V}{\theta L}$

where $V$ is the applied voltage between electrodes and $\theta$ is the angle between the tangent through diaphragm. $L$ is expressed as

$L=\frac{d}{\sin \theta}$

Here, $d$ is the separation between the capacitor plates and is equal to $\left(d_{0}-w(x, y)\right)$. Elemental touch mode capacitance can be expressed as

$d C_{\text {touch }}=d C_{t}+d C_{u t}$

Where, $d C_{u t}$, the elemental cavity capacitance is the function of $L$ and $\theta$ thus

$d C_{u t}=\frac{\varepsilon_{0} \cdot d A}{\theta L}=\frac{\varepsilon_{0} \sin \theta \cdot d A}{\theta d}=\frac{\varepsilon_{0} \sin \theta \cdot d x \cdot d y}{\theta d}$

The touch region between the diaphragm and the bottom electrode is assumed to be square in shape. Elemental touch down capacitance, $d C_{t}$ is expressed as

$d C_{t}=\frac{\varepsilon_{0} \varepsilon_{i} \cdot d A}{t}=\frac{\varepsilon_{0} \varepsilon_{i} \cdot d x \cdot d y}{t}$ 


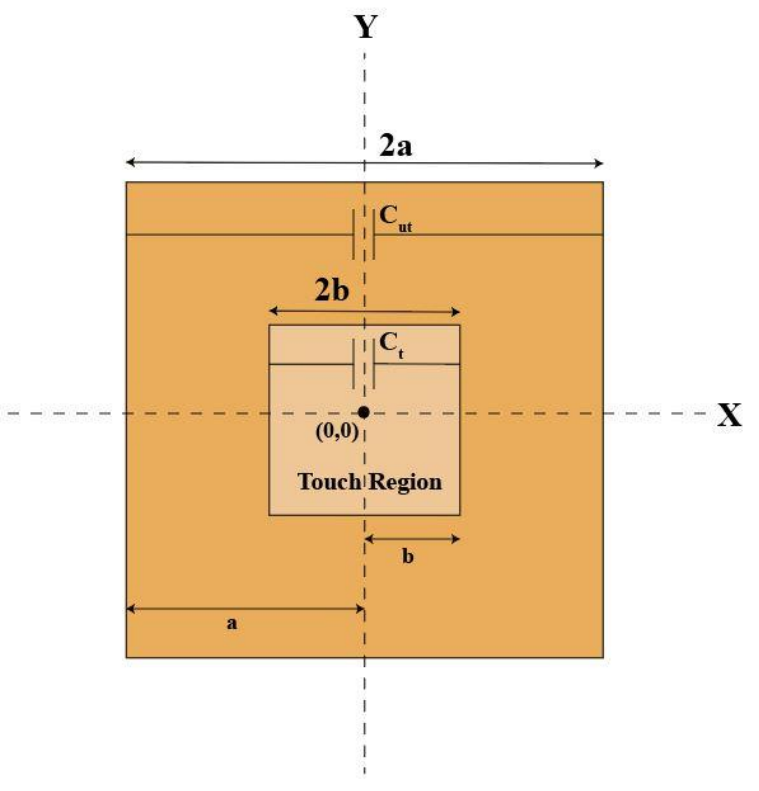

Fig. 5. Top View in Touch Mode

The shaded region in Fig. 5 indicates the touch region. This part contributes to $C_{t}$, the capacitance due to touch region.

$C_{t}=\int_{y=-b}^{b} \int_{x=-b}^{b} \frac{\varepsilon_{0} \varepsilon_{i} \cdot d x \cdot d y}{t}=\frac{\varepsilon}{t} x_{-b}^{b} y_{-b}^{b}=\frac{4 b^{2} \varepsilon_{0} \varepsilon_{i}}{t}$

$C_{u t}$ emerges out of the remaining region apart from grey shaded area in Fig. 5. Hence, solving for $C_{u t}$,

$$
C_{u t}=2 \int_{-a b}^{a} \int_{0}^{a} \frac{\varepsilon_{0} \sin \theta}{\theta d} . d x . d y+2 \int_{b}^{a} \int_{-b}^{b} \frac{\varepsilon_{0} \sin \theta}{\theta d} . d x . d y
$$

where $b$ is half length for the touch region. From Fig. 5 it can be observed that at a point $(b, b)$ deflection of the diaphragm is $d_{0}$. Substituting $x=b, y=b$ and $w(b, b)=d_{0}$ in (1) ,b can be evaluated as

$b=a \sqrt{1-\sqrt[4]{\left(\frac{47 D d_{0}}{P a^{4}}\right)}}$

Substituting $d=d_{0}-w(x, y)$ in (20) and using binomial approximation, the equation is modified as

$$
\begin{aligned}
& C_{u t}=\int_{y} \int_{x} \frac{\varepsilon_{0} \sin \theta}{\theta\left(d_{0}-w(x, y)\right)} \cdot d x \cdot d y \\
& =\frac{\varepsilon_{0} \sin \theta}{\theta d_{0}} \int_{y} \int_{x} d x \cdot d y+ \\
& \frac{\varepsilon_{0} \sin \theta}{\theta d_{0}} \int_{y} \int_{x} \frac{P a^{4}}{47 D}\left(\frac{\left(1-\frac{x^{2}}{a^{2}}\right)^{2}\left(1-\frac{y^{2}}{a^{2}}\right)^{2}}{d_{0}}\right) \cdot d x . d y
\end{aligned}
$$

The integral in (23) can be further simplified as

$$
C_{u t}=\left(C_{1}+C_{2}\right)
$$

As depicted in Fig. 5 for the integral $C_{l}$, the limits for $x$ will be from $b$ to $a$ and similarly, the limits for $y$ will be from - $a$ to $a$.

$$
\begin{aligned}
& C_{1}=\frac{2 \varepsilon_{0} \sin \theta}{\theta d_{0}} \int_{y=-a}^{a} \int_{x=b}^{a} d x \cdot d y+\frac{2 \varepsilon_{0} \sin \theta}{\theta d_{0}} \times \\
& \int_{y=-a}^{a} \int_{x=b}^{a} \frac{P a^{4}}{47 D}\left(\frac{\left(1-\frac{x^{2}}{a^{2}}\right)^{2}\left(1-\frac{y^{2}}{a^{2}}\right)^{2}}{d_{0}}\right) \cdot d x \cdot d y \\
& C_{1}=\frac{4 a(a-b) \varepsilon_{0} \sin \theta}{\theta d_{0}}+ \\
& \frac{2 P \varepsilon_{0} \sin \theta}{\theta d_{0} p_{t}} \cdot \frac{16 a}{15}\left(\frac{8 a}{15}-\left(b+\frac{b^{5}}{5 a^{4}}-\frac{2 b^{3}}{3 a^{2}}\right)\right)
\end{aligned}
$$

Similarly, solving for $C_{2}$ with the following limits, $x=-b$ to $b$ and $y=b$ to $a$ (shown in Fig. 5)

$C_{2}=\frac{4 b(a-b) \varepsilon_{0} \sin \theta}{\theta d_{0}}+\frac{2 P \varepsilon_{0} \sin \theta}{\theta d_{0} p_{t}} \times$

$2\left(b+\frac{b^{5}}{5 a^{4}}-\frac{2 b^{3}}{3 a^{2}}\right)\left(\frac{8 a}{15}-\left(b+\frac{b^{5}}{5 a^{4}}-\frac{2 b^{3}}{3 a^{2}}\right)\right)$

Assuming,

$$
\begin{aligned}
& K=\left(b+\frac{b^{5}}{5 a^{4}}-\frac{2 b^{3}}{3 a^{2}}\right) \\
& C_{r}=\frac{2 \varepsilon_{0} \sin \theta}{\theta d_{0}}
\end{aligned}
$$

Adding $C_{1}$ and $C_{2}$ and substituting (28) and (29) in the sum, $C_{u t}$ is obtained as

$C_{u t}=2 C_{r}\left(a^{2}-b^{2}\right)+2 C_{r} \frac{P}{p_{t}}\left(\frac{64 a^{2}}{225}-K^{2}\right)$

From (19) and (30), $C_{\text {touch }}$ can be mathematically written as,

$$
\begin{aligned}
& C_{\text {touch }}=C_{t}+C_{u t}=\frac{4 b^{2} \varepsilon_{0} \varepsilon_{i}}{t}+2 C_{r}\left(a^{2}-b^{2}\right)+ \\
& 2 C_{r} \frac{P}{p_{t}}\left(\frac{64 a^{2}}{225}-K^{2}\right)
\end{aligned}
$$

\subsection{Capacitive Sensitivity for touch mode operation}

Capacitive Sensitivity is defined as change in capacitance for a given change is the pressure applied to the diaphragm. It can be mathematically represented as

$S=\frac{\partial C}{\partial P}=\frac{\partial C_{t}}{\partial P}+\frac{\partial C_{u t}}{\partial P}$ 
Substituting the values of $C_{t}$ and $C_{u t}$ in (32) from (19) and (30) respectively

$$
\begin{aligned}
& \frac{\partial C_{t}}{\partial P}=\frac{\varepsilon_{0} \varepsilon_{i} a^{2}}{t} \sqrt[4]{\frac{p_{t}}{P^{5}}} \\
& \frac{\partial C_{u t}}{\partial P}=2 C_{r}\left(-\frac{a^{2}}{4} \sqrt[4]{\frac{p_{t}}{P^{5}}}+\frac{1}{p_{t}}\left(\frac{64 a^{2}}{225}-K^{2}\right)+\frac{P}{p_{t}} \cdot\left(-2 K \cdot K^{\prime}\right)\right)
\end{aligned}
$$

Where,

$$
K^{\prime}=\frac{a^{2}}{8 b} \sqrt[4]{\frac{p_{t}}{P^{5}}}\left(1+\frac{b^{4}}{a^{4}}-2 \frac{b^{2}}{a^{2}}\right)
$$

\subsection{Mechanical Sensitivity}

The difference in deflection for a given change in pressure is known as mechanical sensitivity.

$$
S_{m e c}=\frac{\partial w}{\partial P}
$$

Substitute $x=0$ and $y=0$ in (1), $S_{m e c}$ is obtained as

$$
\begin{aligned}
S_{m e c} & =\frac{\partial}{\partial P}\left(\frac{P}{47 D a^{4}}\left(\left(-a^{2}\right)^{2}\left(-a^{2}\right)^{2}\right)\right) \\
S_{m e c} & =\frac{a^{4}}{47 D}
\end{aligned}
$$

\section{DESIGN SPECIFICATIONS AND CONSIDERATIONS}

The MATLAB simulation produced in Fig. (6 to 11) are based on parameters specified in Table I. It is evident from Table I,that the sensor works in Normal Mode for the pressure range $(0-0.023 \mathrm{MPa})$ and operates in Touch Mode for the pressure range $(0.5-3 \mathrm{MPa})$. The highly non-linear transition region between these two modes is observed in the pressure range $(0.023-0.5 \mathrm{MPa})$. In order to validate and compare the results of this analytical approach, values from the research conducted in ${ }^{[1]}$ have been used and the comparison is presented in Fig. (12 and 13).

TABLE I.

DETAILS OF PARAMETERS USED AND THE CHOSEN DESIGN VALUES

\begin{tabular}{lr} 
Parameters & Design values \\
\hline Young's modulus $(E)$ & $170 \times 10^{9} \mathrm{~N} / \mathrm{m}^{2}$ \\
Diaphragm thickness $(h)$ & $5 \times 10^{-6} \mathrm{~m}$ \\
Half-length of diaphragm $(a)$ & $300 \times 10^{-6} \mathrm{~m}$ \\
Poisson's ratio for silicon $(v)$ & 0.28 \\
Permittivity of vacuum $\left(\xi_{o}\right)$ & $8.854 \times 10^{-12} \mathrm{~F} / \mathrm{m}$ \\
Dielectric constant of SiO2 $\left(\xi_{i}\right)$ & 3.7 \\
Dielectric constant of cavity/air & 1 \\
Gap depth $\left(\mathrm{d}_{\mathrm{o}}\right)$ & $2 \times 10^{-6} \mathrm{~m}$ \\
Thickness of insulation layer $(\mathrm{t})$ & $0.1 \times 10^{-6} \mathrm{~m}$ \\
Pressure range in normal mode & $0-0.023 \mathrm{MPa}$ \\
Pressure range in transition mode & $0.023-0.5 \mathrm{MPa}$ \\
Pressure range in touch mode & $0.5-3 \mathrm{Mpa}$ \\
\hline
\end{tabular}

\section{RESULTS AND DISCUSSIONS}

Normal and Touch mode operation are elucidated in subsection 5.1 and 5.2 respectively. The motivation behind the choice of magnitude of the parameters involved in sensor design has been illustrated in subsection 5.3, 5.4, 5.5 and 5.6. Comparison between the sensor performance for circular diaphragm $\mathrm{CPS}^{[1]}$ and square diaphragm CPS has been detailed in subsection 5.7 and 5.8.

\subsection{Capacitance variation with pressure in normal mode}

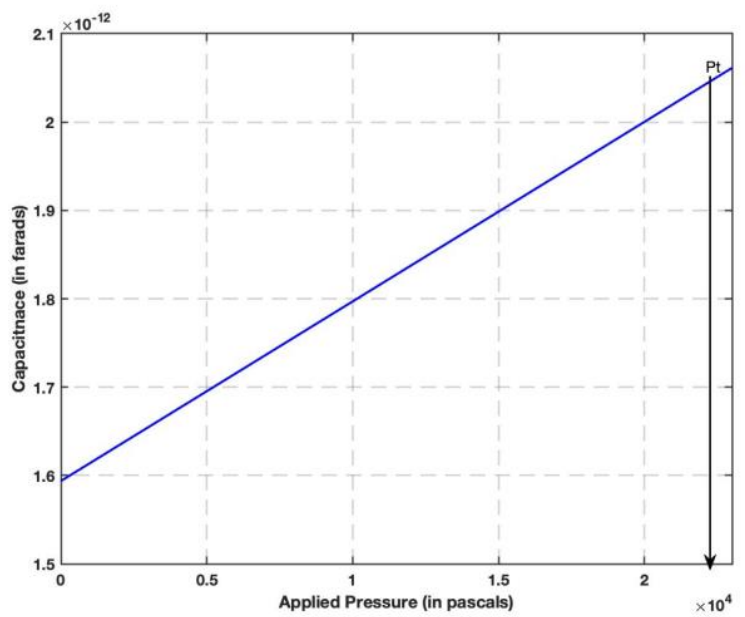

Fig. 6. Capacitance variation with pressure in normal mode

Variation in capacitance with applied pressure for normal mode operation is depicted in Fig. 6. It can be observed that the capacitance varies linearly within the pressure range $(0$ $0.023 \mathrm{MPa})$. The maximum capacitance variation is $2.1 \mathrm{pF}$ for this mode. Fig. 6 also depicts touchpoint pressure $\left(P_{t}\right)$. The transition mode exists during the switch from normal mode to touch mode which can be observed in fig. 7. Because the sensing diaphragm begins to contact the bottom electrode, transition mode is extremely non-linear. The pressure range for the transition region is $(0.023-0.5 \mathrm{MPa})$.

\subsection{Capacitance variation with pressure in touch mode}

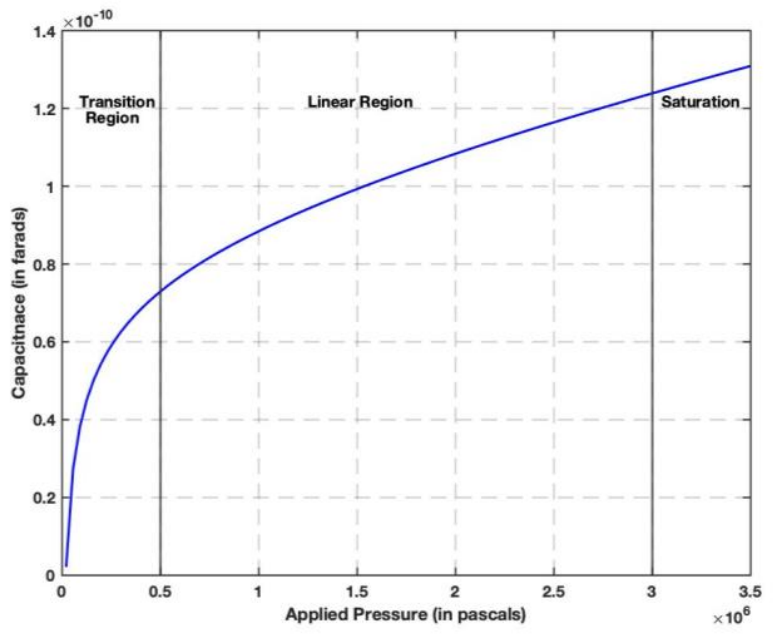

Fig. 7. Capacitance variation with pressure in touch mode 
Fig. 7 indicates capacitance variation with applied pressure for touch mode operation within the pressure range $(0.5$ $3 \mathrm{MPa}$ ). Touch mode can be further divided into two subregions (1) Linear and (2) Saturation. The linear region forms the basis of touch mode sensor operation, as it can be accurately calibrated in terms of pressure. In the saturation region, the diaphragm completely touches the bottom electrode, hence no further change in capacitance is observed. Fig. 6 and 7 indicate that the capacitance developed in touch mode is larger than that in the normal mode of operation. Higher sensitivity and ability to withstand large overload pressure are indicators of touch mode's superior performance.

\subsection{Variation in capacitive sensitivity with half-length of diaphragm for touch mode operation}

The capacitive sensitivity is the fundamental variable of comparison to indicate the performance of a sensor. For $P=1.0 \mathrm{MPa}$, Fig. 8 examines the capacitive sensitivity variation with half-length of diaphragm for touch mode operation. It indicates a negligible variation in capacitive sensitivity for $(0-250 \mu \mathrm{m})$ with a sudden increase after $(300 \mu \mathrm{m})$. Even though capacitive sensitivity is further enhanced with increase in half-length of diaphragm beyond $(300 \mu \mathrm{m})$, there is a reduction in the linear operating range of the sensor. Considering the trade-off between sensitivity and linear range , $a=300 \mu \mathrm{m}$ has been selected for the sensor design as it offers higher sensitivity without compromising the linear operating range.

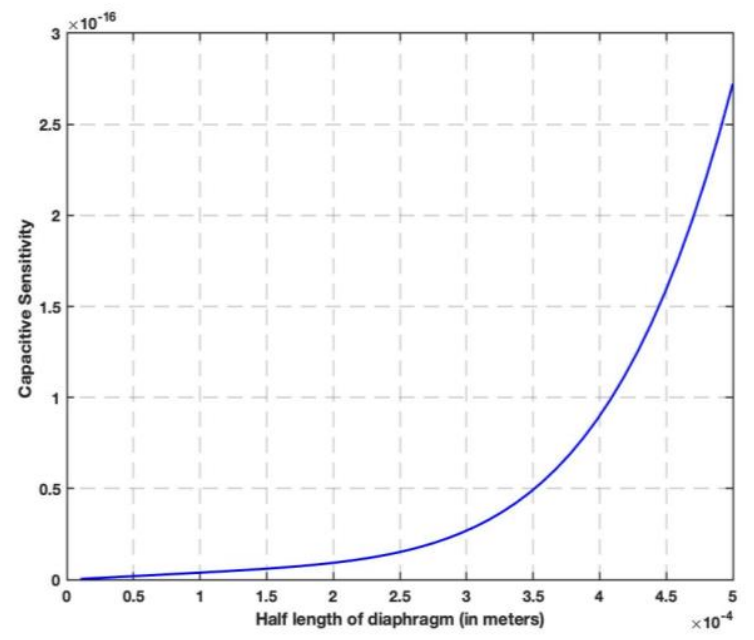

Fig. 8. Capacitance sensitivity vs half-length in touch mode

\subsection{Variation in capacitive sensitivity with gap depth for touch mode operation}

For $P=1.0 \mathrm{MPa}$, Fig. 9 illustrates the capacitive sensitivity variation with gap-depth for touch mode operation. A significant decrease in capacitive sensitivity is observed after a gap depth of $0.5 \mu \mathrm{m}$. The design presented in this paper has a gap depth of $2 \mu \mathrm{m}$ which acts as the limiting value for optimum sensor operation.

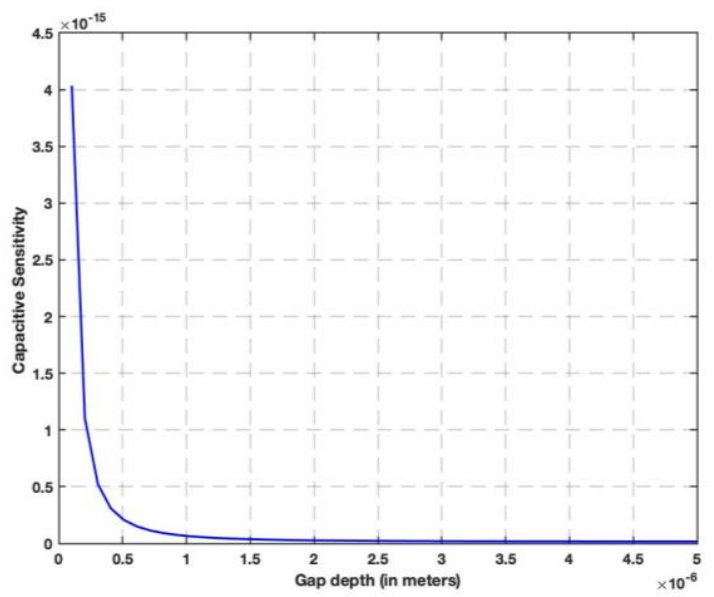

Fig. 9. Capacitance sensitivity vs gap-depth in touch mode

5.5 Variation in capacitive sensitivity with applied pressure for touch mode operation

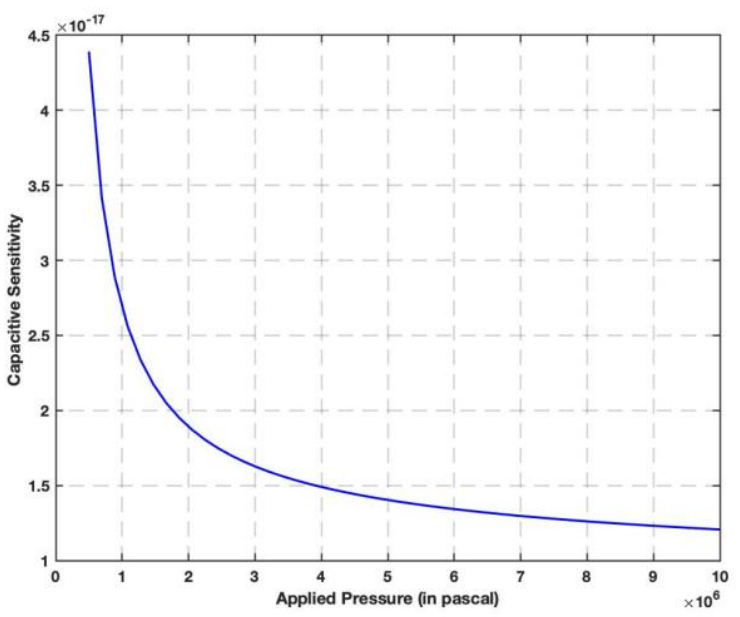

Fig. 10. Capacitance sensitivity vs applied pressure in touch mode

Variation of capacitive sensitivity with applied pressure for touch mode operation is presented in Fig. 10. The capacitive sensitivity decreases with increase in applied pressure and the variation with pressure is almost constant after $3 \mathrm{MPa}$. In the pressure range between $(0.023-0.5 \mathrm{MPa})$, the value of captative sensitivity is considerably large, and this demonstrates the high non-linearity of the transition region. As depicted in Fig. 10, linear operating range for this design is $(0.5-3 \mathrm{MPa})$ and the sensor enters the saturation region after $3 \mathrm{MPa}$ where the capacitive sensitivity is significantly reduced. These observations are also verified by Fig. 7 .

\subsection{Mechanical sensitivity}

The difference of mechanical sensitivity with diaphragm thickness is seen in Figure 11. Due to decreased diaphragm deflection, increasing diaphragm thickness decreases mechanical sensitivity.

It can be concluded that for the given design parameters (Table I), the mechanical sensitivity decreases significantly as the thickness is increased above $5 \mu \mathrm{m}$. As a result, the diaphragm thickness is limited to $5 \mu \mathrm{m} .{ }^{[1]}$. 


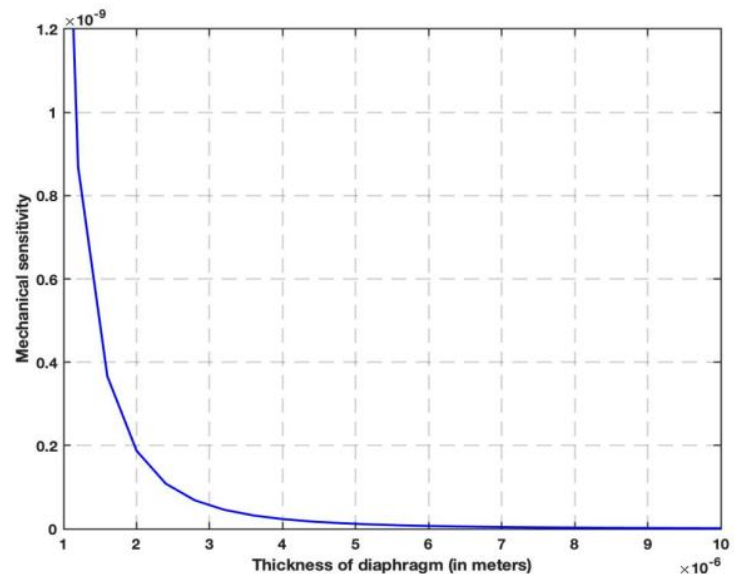

Fig. 11. Variation of mechanical sensitivity with diaphragm thickness

\subsection{Comparative analysis between square and circular diaphragms in normal mode operation}

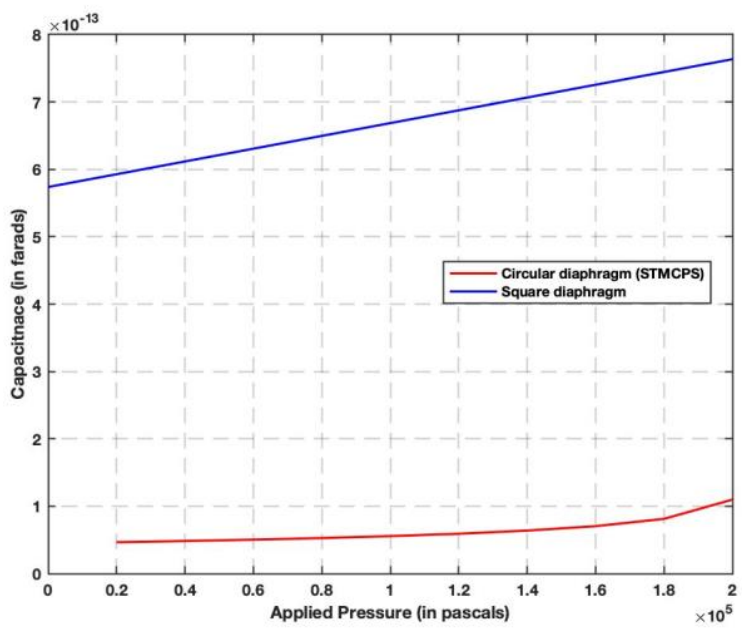

Fig. 12. Comparative plot between square diaphragm CPS and circular diaphragm CPS (Normal Mode)

Fig.12 presents the capacitance variation with applied pressure in normal mode operation for both square and circular diaphragm CPS. It can be inferred that the capacitance, capacitive sensitivity, and full pressure range of normal mode operation is higher for square diaphragms.

\subsection{Comparative analysis between square and circular diaphragms in touch mode operation}

Fig. 13 illustrates the capacitance variation with applied pressure in touch mode operation for both square and circular diaphragm CPS. A square diaphragm occupies a larger area in comparison to a circler diaphragm with the same half-length ( $r=a$, where $r$ is the radius of the circle and $2 a$, is the square's side length). As an increase in the area leads to an increase in capacitance $\left(C=\varepsilon_{0} A / d\right)$, hence higher capacitance and sensitivity are achievable with a square diaphragm CPS which is substantiated by Fig. 13. The results obtained and the interpretations have been summarized in Table II

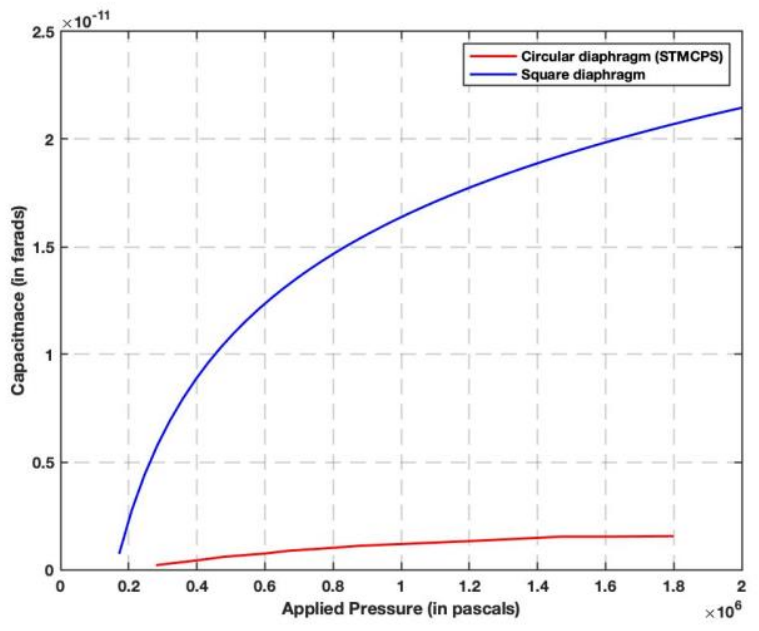

Fig. 13. Comparative plot between square diaphragm CPS and circular diaphragm CPS (Touch Mode)

TABLE II.

COMPARATIVE TABLE FOR CIRCULAR DIAPHRAGM CPS (STMCPS) AND SQUARE DIAPHRAGM CPS IN TOUCH MODE OPERATION

\begin{tabular}{cccc}
\hline $\begin{array}{c}\text { Diaphragm } \\
\text { Geometry }\end{array}$ & $\begin{array}{c}\text { Capacitance } \\
\text { developed (pF) } \\
\text { for applied } \\
\text { pressure of 1.2 } \\
\text { MPa }\end{array}$ & $\begin{array}{c}\text { Capacitance } \\
\text { developed (pF) } \\
\text { for applied } \\
\text { pressure of 1.8 } \\
\text { MPa }\end{array}$ & $\begin{array}{c}\text { Comment on } \\
\text { sensitivity }\end{array}$ \\
\hline Circular[1] & 1.33 & 1.56 & $\begin{array}{c}\text { For the same } \\
\text { pressure range, the } \\
\text { capacitance change } \\
\text { is higher for square } \\
\text { diaphragm CPS. } \\
\text { Sence it can be }\end{array}$ \\
Square & 17.8 & 20.7 & $\begin{array}{c}\text { Herpreted that the } \\
\text { intensitivity of } \\
\text { [proposed }\end{array}$ \\
work] & & $\begin{array}{c}\text { Square diaphragm } \\
\text { CPS is higher than } \\
\text { that of Circular } \\
\text { diaphragm CPS }\end{array}$ \\
\hline
\end{tabular}

\section{Conclusions}

This study presents an analytical model for the determination of normal and touch mode operation of a capacitive pressure sensor with a clamped square diaphragm. The precise study of these modes of operation is crucial for determining the capacitive pressure transducer's operating range and stability. Capacitive and mechanical sensitivity, for example, are derived parameters that provide useful information for sensor design. A justification for the sensor dimensions for optimum operation has been provided to further validate the presented analysis. Finite element method tools enable accurate prediction and modeling of normal and touch mode capacitance. These tools' resource and time complexity, on the other hand, makes their use less practical. This analytical method offers significant ease of computation and clearly examines the higher sensitivity resulting out of the square geometry of the diaphragm. The examined theory and simulated results fully validate the superiority of square diaphragms in terms of ease of manufacturing, higher sensitivity, increased capacitance, and robustness. 


\section{AUTHOR DECLARATIONS SECTION}

* Ethics approval and consent to participate: Not Applicable

* Consent for publication: Yes

* Availability of data and materials: Not Applicable

* Competing interests: Not Applicable

* Funding: Not Applicable

* Authors' contributions: All authors contributed to the study conception and design. Material preparation, simulation and analysis were performed by all the authors together. The first draft of the manuscript was written by Mr. Sumit Kumar Jindal and all authors commented on previous versions of the manuscript. All authors read and approved the final manuscript.

* Acknowledgements: Not Applicable

Compliance with Ethical Standards section

* Disclosure potential conflicts of interest: Not Applicable

* Research involving Human Participants and/or Animals: Not

Applicable

* Informed consent: Not Applicable

\section{REFERENCES}

[1] Jindal, S. K., Mahajan, A., \& Raghuwanshi, S. K.. A complete analytical model for clamped edge circular diaphragm non-touch and touch mode capacitive pressure sensor. Microsystem Technologies, 22(5), 1143-1150, (2016).

[2] Jindal, S. K., \& Raghuwanshi, S. K.. Capacitance and sensitivity calculation of double touch mode capacitive pressure sensor: theoretical modelling and simulation. Microsystem Technologies, 23(1), 135-142, (2017).

[3] Jindal, S. K., Varma, M. A., \& Thukral, D.. Comprehensive assessment of MEMS double touch mode capacitive pressure sensor on utilization of $\mathrm{SiC}$ film as primary sensing element: Mathematical modelling and numerical simulation. Microelectronics Journal, 73, 30-36, (2018).

[4] Varma, M. A., Thukral, D., \& Jindal, S. K.. Investigation of the influence of double-sided diaphragm on performance of capacitance and sensitivity of touch mode capacitive pressure sensor: numerical modeling and simulation forecasting. Journal of Computational Electronics, 16(3), 987-994, (2017).

[5] Molla-Alipour, M., \& Ganji, B. A.. Analytical analysis of mems capacitive pressure sensor with circular diaphragm under dynamic load using differential transformation method (DTM). Acta Mechanica Solida Sinica, 28(4), 400-408, (2015).

[6] Rochus, V., Wang, B., Tilmans, H. A. C., Chaudhuri, A. R., Helin, P., Severi, S., \& Rottenberg, X.. Fast analytical design of MEMS capacitive pressure sensors with sealed cavities. Mechatronics, 40, 244-250, (2016)

[7] Kang, M. C., Rim, C. S., Pak, Y. T., \& Kim, W. M.. A simple analysis to improve linearity of touch mode capacitive pressure sensor by modifying shape of fixed electrode. Sensors and Actuators A: Physical, 263, 300-304, (2017).

[8] Jindal, S. K., \& Raghuwanshi, S. K.. A complete analytical model for circular diaphragm pressure sensor with freely supported edge. Microsystem Technologies, 21(5), 1073-1079, (2015).

[9] Ganji, B. A., \& SHAMS, N. M.. Modeling of capacitance and sensitivity of a MEMS pressure sensor with clamped square diaphragm, (2013).

[10] Jindal S.K., Mahajan A \& Raghuwanshi S.K.. Reliable beforefabrication forecasting of normal and touch mode MEMS capacitive pressure sensor: Modeling and simulation. Journal of Micro/Nanolithography, MEMS, and MOEMS. 16.1 10.1117/1.JMM.16.4.045001, (2017).

[11] Peterson, K.E. (1982) : Silicon as a mechanical material. Proc. IEEE 70, 420-457 [12] Hsu, T.R.: MEMS and Microsystems Design and Manufacture. Tata Mc-Graw Hill, New York (2002).

[12] Sharma, A., Mittal, M., \& Saini, D.. Virtual Fabrication, 3D Electromechanical Simulation and System Level Modeling of MEMS Capacitive Pressure Sensor for Tire Pressure Monitoring System. Sensors \& Transducers, 143(8), 106, (2012).

[13] Lee, M. K., Eom, J., \& Choi, B.. Numerical analysis of touch mode capacitive pressure sensor using graphical user interface. In Recent Progress in Data Engineering and Internet Technology, Springer, Berlin, Heidelberg (pp. 371-377)., (2013).

[14] J Bergqvist. Finite Element Modelling and characterization of Silicon condenser microphone with a highly perforated backplate. Sens. Actuators Lett. A39, (1993)

[15] Bao, M.. Analysis and design principles of MEMS devices. Elsevier, (2005).

[16] S Timoschenko, Theory of Plates and Shells, Mc-Graw Hill, New York, (2006) 